\title{
«Team Coverage» im Nachwuchsleistungssport
}

\section{EXERCISE IS MEDICINE}

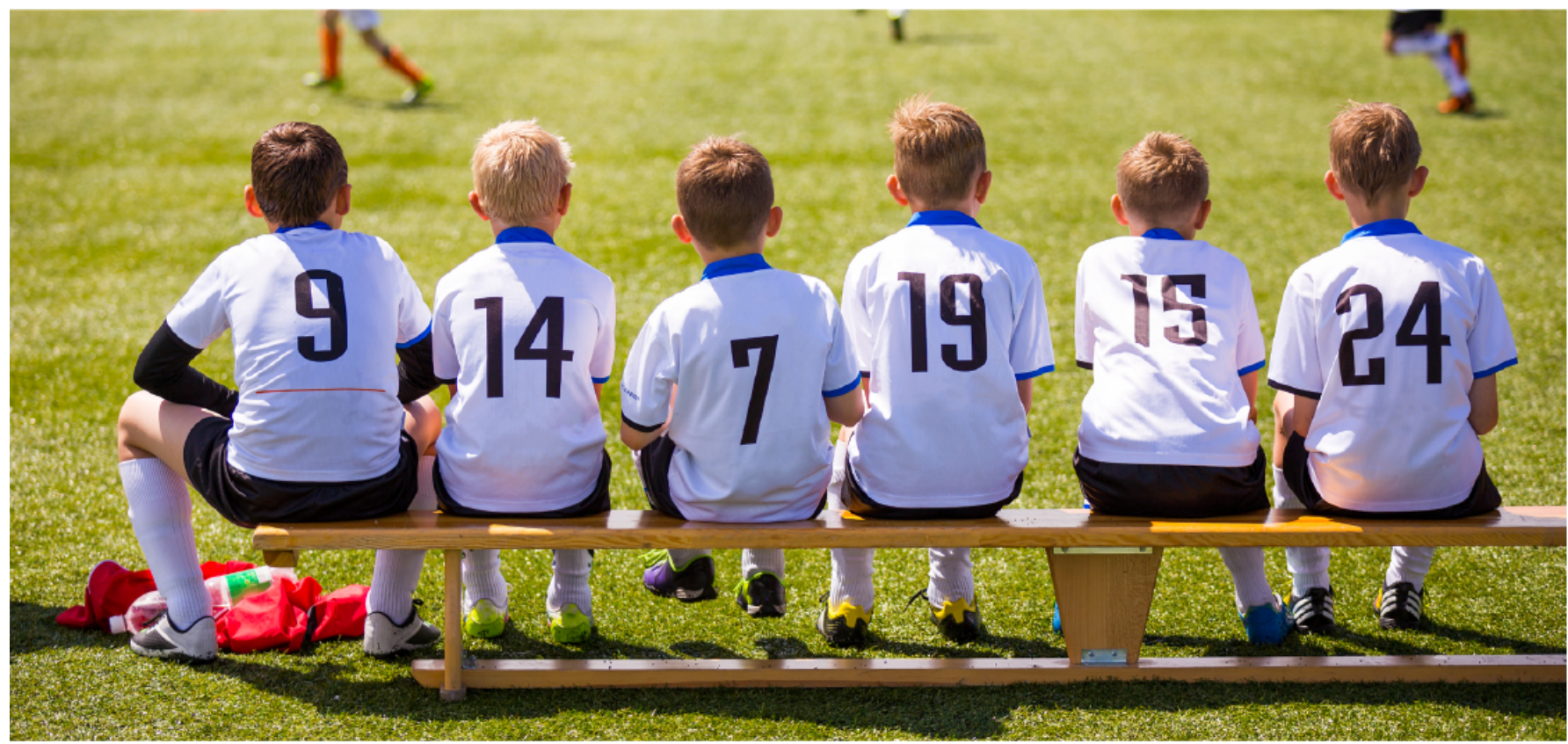

\section{Interview von Patrick Vavken mit Markus Renggli}

Vavken: Worin unterscheidet sich die sportmedizinische Betreuung von Nachwuchsleistungssportlern von derjenigen von Erwachsenen?

Renggli: Im Kinder- und Jugendsport bewegt man sich vorwiegend im nonprofessionellen Umfeld. Meist gibt es noch keine definierten Strukturen in der sportmedizinischen Betreuung der einzelnen Sportarten. So kommen die jungen Athleten meist durch Zuweisung der Haus- und Kinderärzte oder durch Empfehlung der Trainer in meine Sprechstunde. Im Kunstturnen, wo ich für das Leistungszentrum verantwortlich bin, ist dies bei dem hohen Trainingsumfang der noch jungen Sportler und früher Spezialisierung schon professionell organisiert.

Welche besonderen Anforderungen stellen sich im Umgang mit sportmedizinischen Problemen von Nachwuchsleistungssportlern?

Als Sportmediziner ist man in der Betreuung von Nachwuchsleistungssportlern vor allem ein geforderter Kommunikator. MRI-Berichte müssen in altersgerechte Sprache übersetzt werden und auch bezüglich Relevanz gewichtet werden. Das Fachchinesisch von konsiliarisch zugezogenen Orthopäden muss sinnvoll übersetzt werden und Befunde dem Trainerstaff und Eltern mitgeteilt werden. Meist sind es die Eltern, welche 
vor allem wissen möchten, wann denn ihr junger Athlet wieder an Wettkämpfen teilnehmen oder im Mannschaftssport eingesetzt werden kann. Für die jungen Sportler selbst ist der Zeithorizont noch eher auf kurzfristige Ziele ausgerichtet, während das Betreuungsteam langfristig in Olympiazyklen denkt.

\section{Beriekungsgeflecht Nackwachble istunges port}

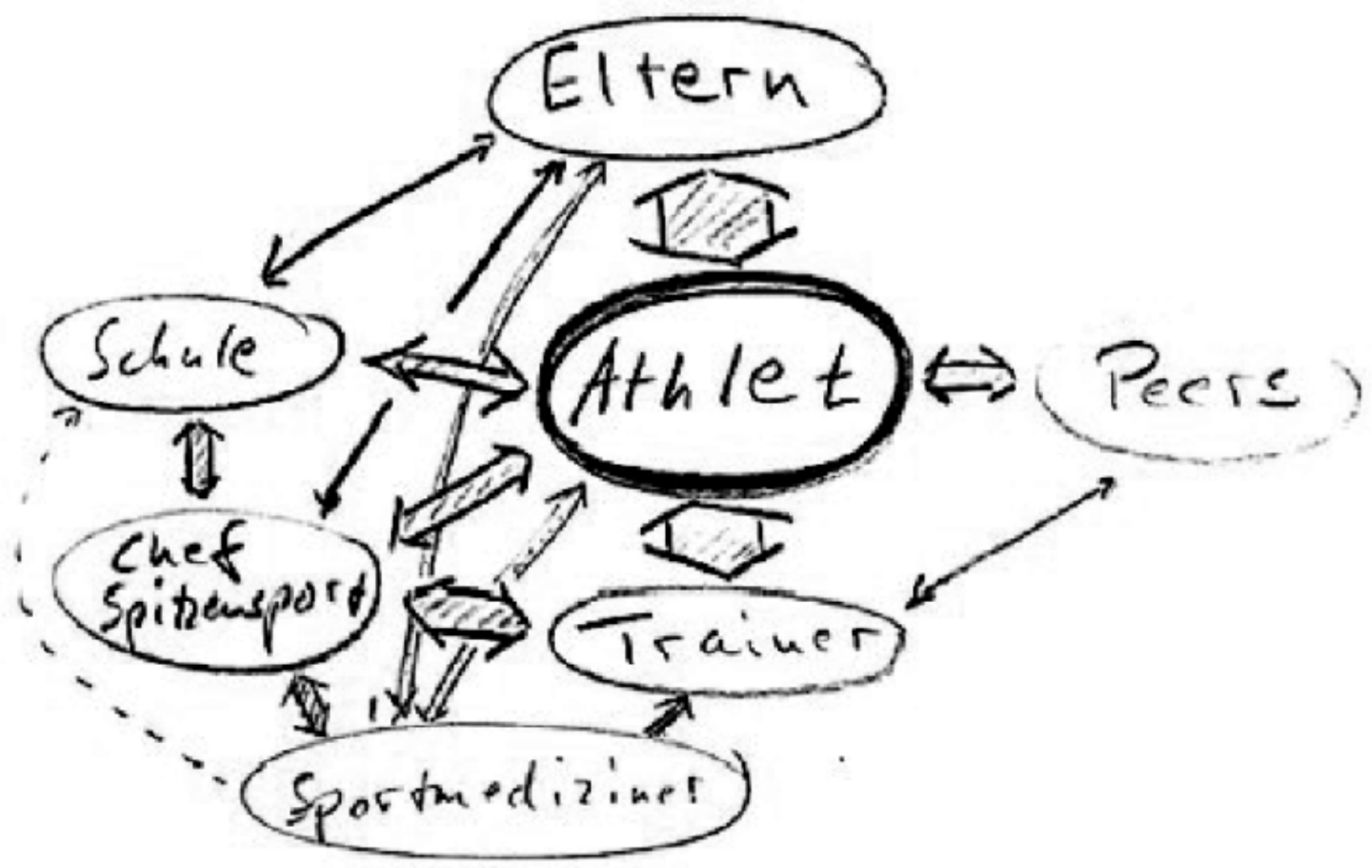

Wo liegen z.B. altersabhängige Besonderheiten vor, welche es im Erwachsenensport so nicht gibt? Im Nachwuchsleistungssport ist der Übergang vom Kind zum pubertierenden Jugendlichen von entscheidender Bedeutung. In vielen Sportarten zeigen sich neue biomechanische Herausforderungen, welche durch die Veränderung der Körperzusammensetzung sowie der Hebelarme infolge Wachstum zu meistern und auch auszuhalten sind. Der dropout vom Sport ist in diesem Altersabschnitt wohl am grössten. In einzelnen Sportarten haben Frühentwickler Vorteile (Leichtathletik), in anderen Spätentwickler (Kunstturnen). Deshalb haben die jährlichen sportmedizinischen Untersuchungen und das Erfassen der Messdaten für die sportmedizinische Begleitung eine entscheidende Bedeutung. Dabei spielt je nachdem die Bestimmung des Knochenalters und der Wachstumsgeschwindigkeit zur Voraussage der körperlichen Entwicklung eine grosse Rolle. So gibt es zum Beispiel im Fussball den sog. Nachteilsausgleich in Form der Carte blanche, welche es Spätentwicklern erlaubt, in der tieferen Alterskategorie weiterzuspielen. 


\section{Aufgaben Team Coverage Nachwuchs- leistungssport}

- Eintrittsuntersuchung neuer Kaderathleten

- Jährliche SPU

- Vernetzung mit Verbandsfunktionären (Chef Spitzensport) und Trainerstaff

- Regelmässige Besuche vor Ort zusammen mit Physio

- Sportmedizinische Betreuung nationaler und internationaler Wettkämpfe mit Notfallkonzept/ Sanität usw.

- Fachvorträge halten für Eltern und Trainer z.B. anlässlich Spitzensportkonferenz

- Kontrolle der Einhaltung der Charta SEMS und Antidoping Schweiz

- Persönliche sportartspezifische Weiterbildung

Wie steht es denn in der Schweiz um die regelmässige SPU im Nachwuchsbereich?

Eine einheitliche Empfehlung für regelmässige sportmedizinische Untersuchungen seitens swissolympic ist meines Wissens fehlend und wird auch im Tarmed nicht abgebildet. Bemühungen diesbezüglich sind aber beim neuen Tarif Tardoc von unserer Fachgesellschaft in Verhandlung. Es ist einzelnen Sportverbänden überlassen, Regelungen zu formulieren. So gibt es einzelne Sportarten, bei welchen ein Untersuch vor Wettkämpfen in bestimmten Leistungsbereichen vorgeschrieben ist, wie z.B. im Rudersport, oder bei Aufnahme in in ein nationales Kader z.B. Eiskunstlauf. Zudem ist auch bei Aufnahme in ein Sportgymnasium oft eine SPU vorgeschrieben.

\section{Wo gibt es bezüglich SPU deiner Ansicht nach dringend Nachholbedarf?}

Gerade in den weitverbreiteten Mannschaftssportarten wie Fussball und Handball mit hohem Verletzungsrisiko, fehlt die regelmässige präventive Untersuchung im Juniorbereich noch weitgehend. Oft sieht man die Jugendlichen erst bei Verletzung oder Overuseproblemen erstmalig. Obwohl schon sehr viel Know-how in der Prävention (Bsp. Fifa 11+) vorhanden wäre, wird die regelmässige Anwendung im Training in der Schweizer Nachwuchstrainerszene noch kaum umgesetzt. 
Eine 14-jährige Handballerin kommt zur SPU, weil sie in die Sportkanti wechselt. Sie wurde jahrelang vom Hausarzt wegen «Anstrengungsasthma» mit Ventolin behandelt. Eine Spirometrie hat nie stattgefunden, und eine Verbesserung der Ausdauerleistung wird nicht festgestellt. Sie gerate rasch ausser Atem und ermüde. Das Sportlabor zeigt eine schwere Anämie mit $\mathrm{Hb} 8.7 \mathrm{~g} / \mathrm{l}$ und ein Ferritin unter 3.

Kannst du Schwerpunkte in der Beraterfunktion des Sportmediziners bei der Betreuung von Teams beispielhaft erläutern?

Bei gravierenden Verletzungen in wichtigen Altersabschnitten vor internationalen Wettkämpfen (z.B. Junioren-Europameisterschaften) spielt der Verbands- oder Teamarzt als Berater eine wichtige Rolle. Er hilft mit, Risiken für die körperliche langfristige Entwicklung gegenüber der Chance einer Wettkampfteilnahme abzuwägen und soll auch auf die Möglichkeit des Scheiterns und der Weichenstellung bezüglich des Einstiegs ins Berufsleben (Lehre, Studium) hinweisen. Anders als bei Elitesportlern spielt das Alter halt doch eine entscheidende Rolle bei der Möglichkeit, sich an Juniorenwettkämpfen messen zu können. Gerade bei sehr trainingsintensiven Sportarten ist es eine Gratwanderung, zwischen Sport, Schule und Transportfahrten genügend Zeit für Mahlzeiten und Regeneration sowie soziale Kontakte mit Peers zu finden. Auch hier hat der Teamarzt eine Berater- und Schulungsfunktion, eventuell in Form von Themenvorträgen für Trainer, Eltern und die jungen Sportler selbst.

Ein 14-jähriger Triathlet hat im Internet gelesen, dass man durch vegane Ernährung seine Leistung verbessern könne. Die Mutter ist besorgt und schleppt den Jüngling gegen seinen Willen in die sportmedizinische Sprechstunde. Selbstredend ist er für sachliche Argumente nicht zugänglich, und auch die Aspekte des Körpers im Wachstum sind für ihn nicht relevant.

Inwiefern haben sich die jüngst in den Medien geschilderten Grenzverletzungen durch Trainer im Umgang mit Turnerinnen in deiner Arbeit als Teamarzt ausgewirkt?

Da ich von Haus auf ja Kinder- und Jugendmediziner bin, ist mir die Sensibilität diesbezüglich schon immer bewusst gewesen. Die Betreuung und Untersuchung von Turnerinnen findet sowohl im Leistungszentrum als auch in der Praxis immer entweder zusammen mit der Physiotherapeutin des medical teams oder in Anwesenheit eines Elternteils statt. Wichtig erscheint mir auch, dass das Arztgeheimnis gegenüber dem Trainerteam strikt eingehalten wird, ausser es liegt im Einverständnis und Interesse des Athleten. Nur so wird man als Vertrauensperson wahrgenommen und erfährt allenfalls etwas über psychische oder physische 
Übergriffe. Es ist übrigens nicht so, dass nur Mädchen betroffen sind, es kommt auch bei Jungs zu respektlosen und verletzenden Verhaltensweisen der Trainer und dies auch in anderen als ästhetischen Sportarten.

\section{Eine 9-jährige Kunstturnerin wird auf Empfehlung der Trai- nerin vorgestellt, weil sie trotz täglicher unzähliger Klimm- züge zu wenig Muskeln entwickle in den Armen. Kann man das durch Nahrungsergänzungsmittel verbessern oder liegt gar ein Mangel vor?}

\section{Inwiefern ist es wichtig, über die verschiedenen Belastungen der Sportarten Kenntnisse zu haben, und welche Quellen gibt es dafür im Nachwuchsleistungssport?}

Es ist meiner Ansicht nach sehr wichtig regelmässig im entsprechenden Leistungszentrum auch vor Ort zu sein und das Gespräch mit Trainern und Athleten zu suchen. Nur so kann man auch nach Unfällen die verletzungsadaptierte, loadgesteuerte Trainingsfreigabe erteilen. Oft wird durch erstbehandelnde Orthopäden schemahaft unspezifisch lange Sportkarenz verordnet. So geht oft viel zu viel Trainingszeit und Wiederaufbauzeit verloren. Gerade Jugendliche brauchen die Integration im Trainingsbetrieb und den Kontakt mit ihren Sportkameraden als Motivation für die Rückkehr zum Leistungssport. Hier sind auch die Trainer gefordert, individuelle Trainingspläne zu erstellen. Einen guten Überblick über die Belastungsprofile und typische Verletzungsmuster im Jugendalter bei verschiedenen Sportarten, findet man auf der Homepage der Gesellschaft für pädiatrische Sportmedizin unter dem Link: https://www.kindersportmedizin.org/download.

\section{Wo siehst du noch «Baustellen» im Bereich des Nachwuchsleistungssports?}

Durch den Schritt an die Medien sind in einigen Sportarten Missstände im Umgang mit den jungen noch vulnerablen Nachwuchssportlern ans Licht gekommen und so wie es aussieht, werden Verbesserungen strukturell vorgenommen. Dies genügt meiner Ansicht nach aber noch nicht. Vieles bleibt noch im Verborgenen, und vieles wird als Preis zum Erfolg in Kauf genommen. Im Nachwuchssport sind viele semiprofessionelle und oft ehrenamtliche Trainer unterwegs ohne genügende Ausbildung im z.Bsp. sportpsychologischen Bereich. Auch mischen gerade im Fussballsport zwielichtige Spielerberater mit, welche unrealistische Versprechungen machen.

Bei Mehrkampfleichtathleten mit verschiedenen Disziplinentrainern ist es äusserst schwierig, in den bestehenden Sportkantonsschulen einen vernünftigen Trainingsplan zu erstellen, da diese vor allem auf Mannschaftssportarten ausgerichtet sind.

Des Weiteren beobachte ich, dass beim Ausscheiden eines Leistungssportlers, sei es verletzungsbedingt oder mangels Qualifikation ins oberste Kader, noch ungenügende Konzepte fürs Detraining und psychologische Begleitung bestehen. Die Sportler fühlen sich vom Verband fallen gelassen und eine Nachbetreuung fehlt. 
Wenn man als Teil eines ganzen Räderwerks in der Poleposition an einem nationalen oder internationalen Wettkampf die sportlichen Erfolge seiner langjährigen Schützlinge hautnah erleben darf und gleichzeitig einen sportmedizinischen Beitrag in der Wettkampforganisation leisten kann, zahlt sich das Engagement immer aus.

\section{Korrespondenzadresse}

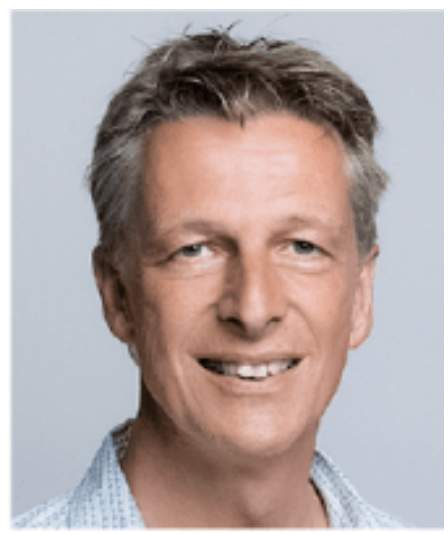

Markus Renggli

sportmedAKTIV

5413 Birmenstorf

info@sportmedaktiv.ch 